\title{
RICH photon detector module based on Geiger-mode APDs
}

\author{
Samo Korpar* \\ Faculty of Chemistry and Chemical Engineering, University of Maribor, \\ and Jožef Stefan Institute, Ljubljana, Slovenia \\ E-mail: 'samo. korpar@ijj.

\section{Hassan Chagani} \\ Jožef Stefan Institute, Ljubljana, Slovenia \\ E-mail: 'thassan. chagani@ijos. Sij'
}

\section{Rok Dolenec}

Jožef Stefan Institute, Ljubljana, Slovenia

E-mail: 'rok. dolienec@ijos.sì

\section{Peter Križan}

Faculty of Mathematics and Physics, University of Ljubljana,

and Jožef Stefan Institute, Ljubljana, Slovenia

E-mail: peter.krizan@ija.sín

\section{Rok Pestotnik}

Jožef Stefan Institute, Ljubljana, Slovenia

E-mail: 'rok.pestotnik@ijs. sì'

\section{Aleš Stanovnik}

Faculty of Electrical Engineering, University of Ljubljana,

Jožef Stefan Institute, Ljubljana, Slovenia

E-mail: 'ales. stanovnik@ija.sì

The possibilities offered by Geiger-mode APDs, also known as silicon photomultipliers, for detection of Cherenkov photons emitted by pions in an aerogel radiator, have been investigated. The main issue is the detection of single photons in the relatively high background of noise pulses. A suitably narrow coincidence time window eliminates most of this background, while the use of light concentrators increases the acceptance, i.e. the sensitivie surface over which Cherenkov photons are collected. With such techniques, we have measured a number corresponding to 3.7 detected photons per ring. However, a thicker radiator and other improvements of the experimental apparatus, might increase this figure for a factor of 10. Such a ring imaging Cherenkov detector would then meet the requirements for particle identification inside the upgraded Belle spectrometer.

International Workshop on New Photon Detectors PD09

June 24-26 2009

Shinshu University Matsumoto Japan

\footnotetext{
* Speaker.
} 


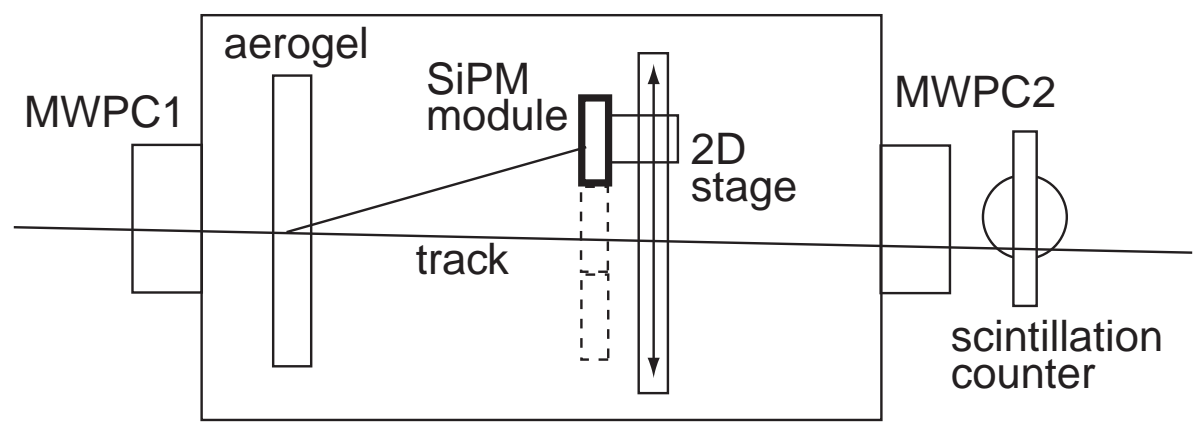

Figure 1: Schematic figure of the experimental apparatus.

\section{Introduction}

One requirement of the particle identification system for the upgraded Belle detector [i1, is that it should provide a $4 \sigma$ separation of pions from kaons up to $4 \mathrm{GeV} / \mathrm{c}$. Because of limited space, it seems that a proximity focusing ring imaging Cherenkov detector, with aerogel as radiator, is the best choice. Different photon detectors have been tested with respect to efficiency, position resolution, timing resolution and other parameters $\left[\begin{array}{l}1 \\ 3\end{array}\right]$. However, none of these is sufficiently insensitive to the high magnetic field $(1.5 \mathrm{~T})$ present inside the Belle spectrometer. The recent appearance on the market of the Geiger-mode APDs, known also as silicon photomultipliers

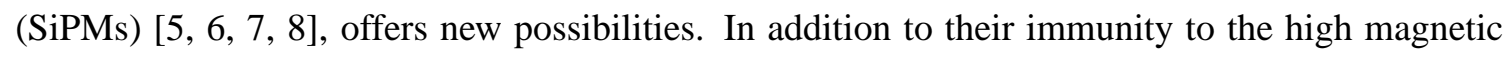
fields, such photo detectors are robust, compact, require low operation voltages and have relatively high quantum efficiency for detection of visible light. The downside is the not very high fraction of active area $(10 \%-20 \%)$ and the high dark count rate $\left(1 \mathrm{MHz} / \mathrm{mm}^{2}\right)$. In order to study the possibility of detection of single Cherenkov photons and the feasibility of a SiPM based Cherenkov ring imaging detector, we have constructed a module consisting of $8 \times 8$ SiPMs, with which we have performed measurements of Cherenkov photons emitted by $120 \mathrm{GeV} / \mathrm{c}$ pions in an aerogel radiator. The second section briefly describes the experimental apparatus, the third section contains the results, which is followed by a short discussion.

\section{Experimental Set-up}

The experimental apparatus is shown schematically in Fig. $i_{-i}^{i}$ Incident pions are triggered by scintillation counter and their tracks are registered with multiwire proportional chambers (MWPCs). In the $1 \mathrm{~cm}$ thick aerogel radiator, with refractive index $\mathrm{n}=1.03$, the $120 \mathrm{GeV} / \mathrm{c} \mathrm{pi}$ ons radiate Cherenkov photons at an angle of about $240 \mathrm{mrad}$, with respect to the track direction. These photons are detected by a position sensitive photon detector, consisiting of an array of $8 \times 8$

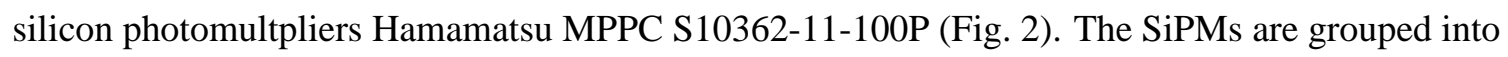
pads of $5.08 \mathrm{~mm} \times 5.08 \mathrm{~mm}$, consisting of $2 \times 2$ SiPMs. Each SiPM has a sensitive area of $1 \mathrm{~mm}^{2}$, which means that the geometrical coverage is only $15.5 \%$. The 4 SiPMs of one pad are connected to one electronic channel, the pulse of which is registered by a multi-hit TDC. The time distribution of pulses is shown in Fig. inti $_{i}$ It is obvious that the background, i.e. the noise rate is high, but that it is generally not in coincidence with the Cherenkov pulses. 

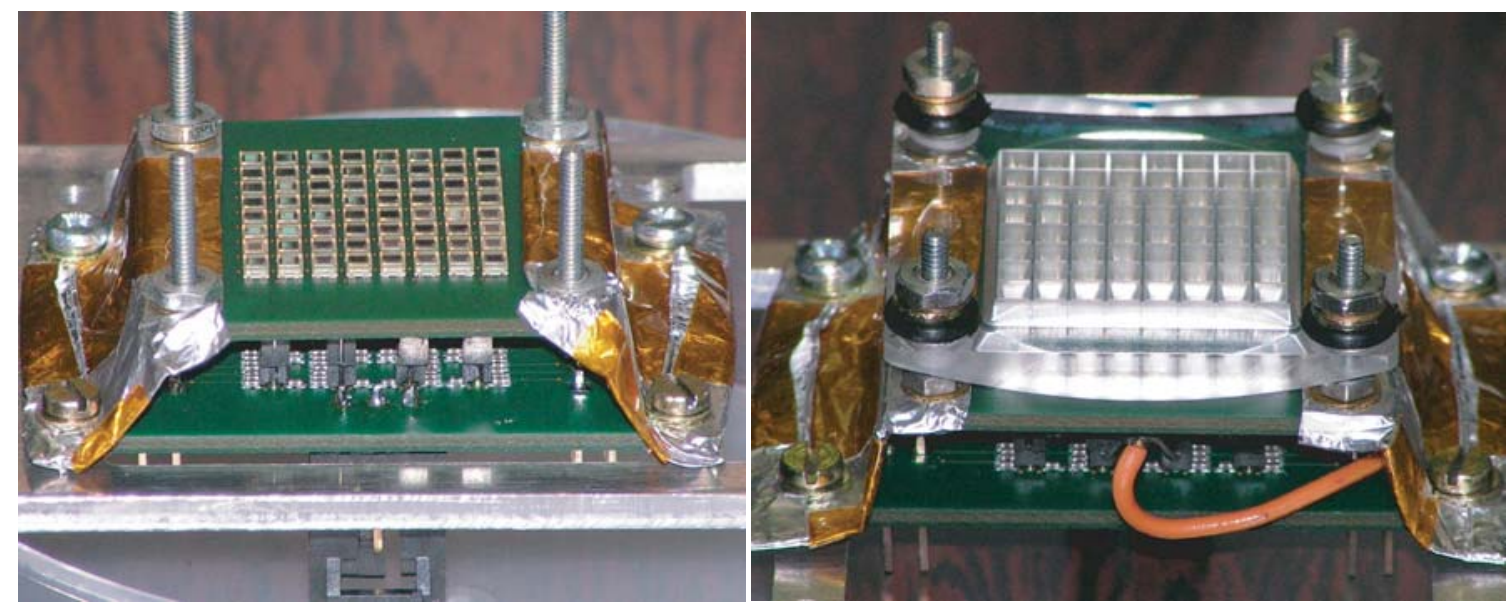

Figure 2: The photon detector module consisting of 64 SiPMs without (left) and with (right) the pyramidal light guides. Four SiPMs are grouped into a single read-out channel.

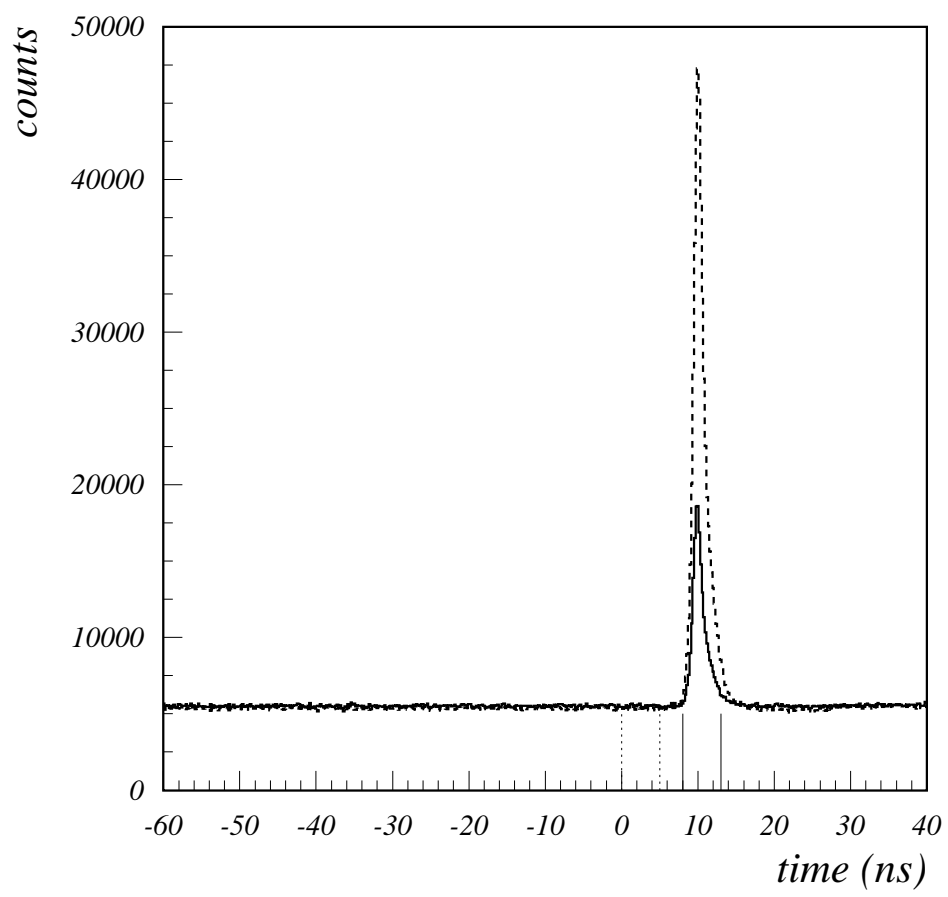

Figure 3: The time spectra for the SiPM pulses in the case without (solid) and with (dashed) the light collection system. The indicated cuts correspond to a $5 \mathrm{~ns}$ time windows.

A possible improvement of such a photon detector would be to increase the fraction of sensitive area (the geometrical coverage or acceptance), which would increase the signal-to-noise ratio. This has been attempted with the aid of light guides, i.e. light collectors in the form of truncated pyramids. The entrance window is a square of $2.54 \mathrm{~mm} \times 2.54 \mathrm{~mm}$ and the exit window is $1 \mathrm{~mm} \times 1 \mathrm{~mm}$. The light guide is $4 \mathrm{~mm}$ long, resulting in a $10^{\circ}$ inclination of the lateral surfaces with respect to the light guide axis. The light guides have been machined out of UV-transparent perspex, used for the lens system of the HERA-B RICH [י] 


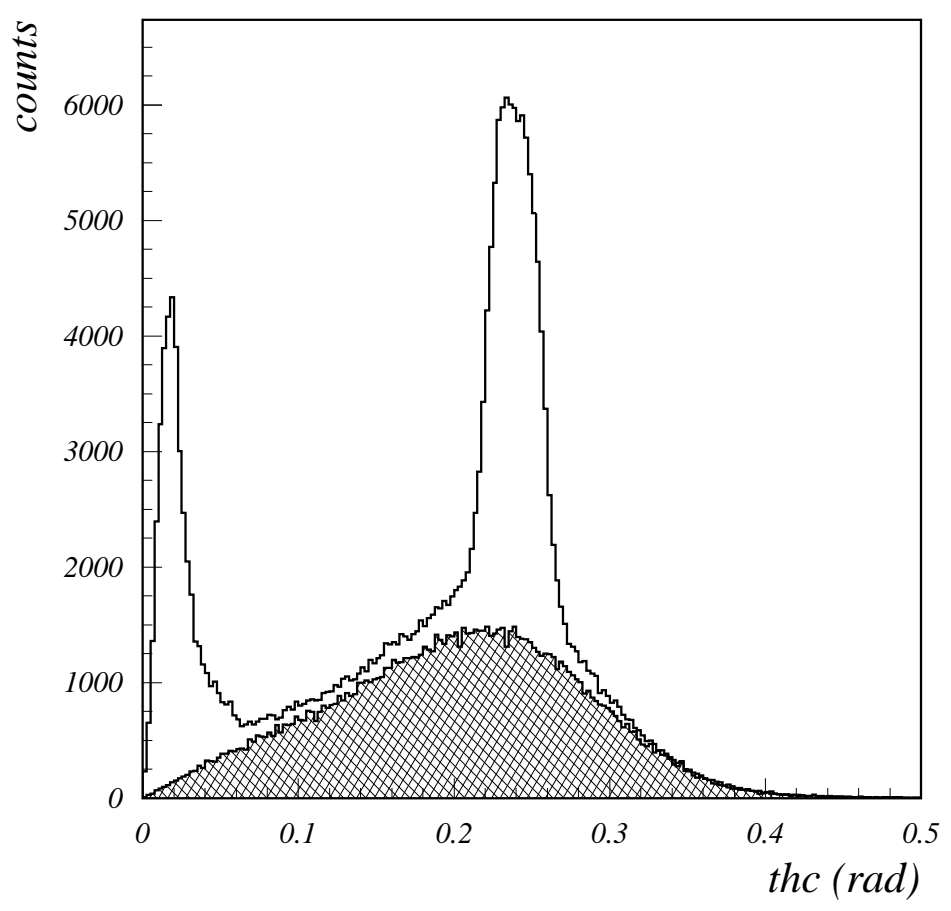

Figure 4: The distribution of SiPM hits with respect to their corresponding Cherenkov angle for pulses inside the $5 \mathrm{~ns}$ time window (open histogram) and for pulses in a corresponding out-of-time window (shaded). The data are from measurements without the light guides.

transmitted by the light guide and hitting the sensitive area of the SiPM, has been calculated by Monte Carlo techniques and found to be $65 \%$. The $35 \%$ loss occurs mostly in the $0.3 \mathrm{~mm}$ gap between the light guide exit window and the SiPM sensitive volume. This loss could partly be due to absorption in the light guide and refraction through the lateral surfaces. The expected acceptance of $65 \%$ represents a substantial improvement with respect to the $15.5 \%$ geometrical coverage. As the module with $64 \mathrm{SiPMs}$ is not large enough to cover the entire Cherenkov ring, measurements had to be made in 9 positions on a $3 \times 3$ grid.

\section{Results}

Measurements have been performed with and without the light guide array in order to evaluate it's effect on the number of detected Cherenkov photons per ring. The increase in the signal-tonoise ratio can be best seen in Fig. $\overline{3}_{-1}$, which shows the time spectrum of SiPM pulses with and without the light guides. The measured improvement for a factor of 2.3 is less than the factor of 3.5 obtained from Monte Carlo calculations of the light guide transmission. This discrepancy could be due to the less-than-perfect overlap of the light guides and SiPMs and to non-specular reflection and refraction on the light guide lateral walls.

The distribution of hits with respect to their corresponding Cherenkov angle is shown in Fig. only for hits within a $5 \mathrm{~ns}$ time interval. For the case that the time window is centered on the peak of the distribution in Fig. $\bar{\beta}_{p}^{\prime}$ one observes also a peak in the distribution with respect to the Cherenkov angle. The Cherenkov angle distribution for hits not in coincidence with the charged particle, the 

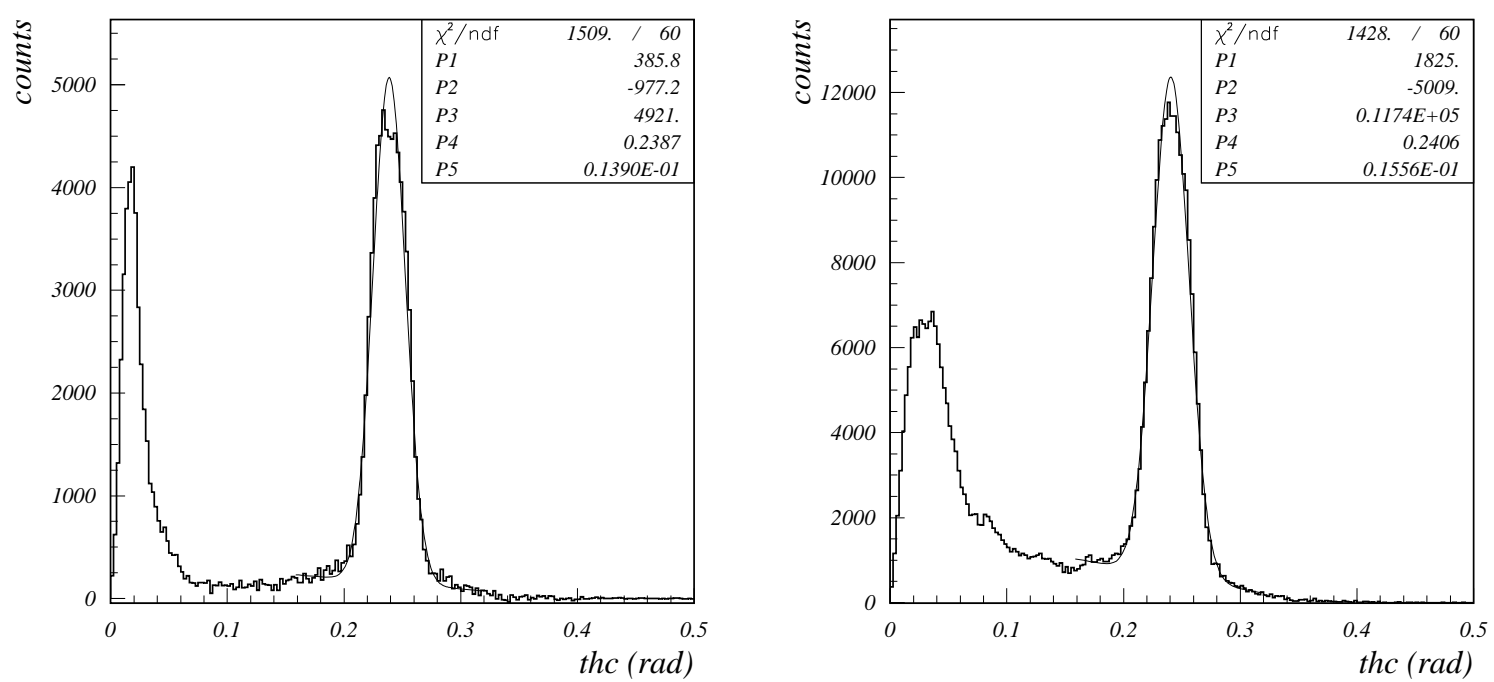

Figure 5: The distribution of hits in Cherenkov angle with the out-of-time background subtracted for the case without (left) and with (right) the light guide system.
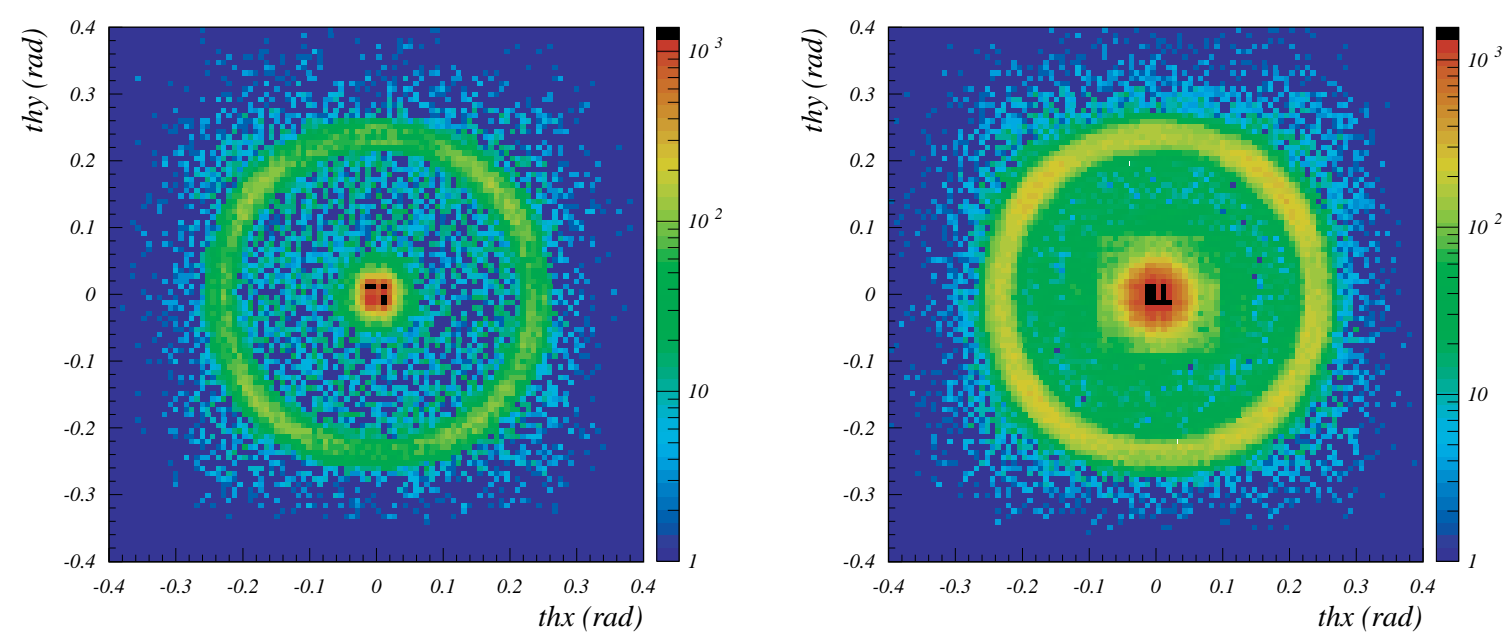

Figure 6: The accumulated SiPM hits represented in Cherenkov angle space for measurements without (left) and with (right) the light collection system. The azimuthal distribution on the Cherenkov ring is uneven due to limitations in acceptance.

background distribution as shown in Fig. 离 is obtained. By subtracting this background and fitting a Gaussian to the Cherenkov photon peak (Fig. photons per incident charged particle. The numbers obtained are 1.6 photons/ring without the light guides and 3.7 photons/ring with the light guides. Accumulated rings in Cherenkov angle space, corresponding to the distributions in Fig. ${ }_{2}^{\bar{s}}$ are shown in Fig. ${ }_{-i}^{\prime} \overline{6}_{i}^{\prime}$

We note that the width of the Cherenkov photo peak of Fig. $\overline{5}_{1}^{\prime}$ corresponds to $\sigma \approx 14 \mathrm{mrad}$ and roughly agrees with estimates based on photon detector pad size, distance from radiator to detector, emission point uncertainty and tracking accuracy. However, the intention of the present study was not to optimize the angular resolution, but to evaluate the attainable photon yield. 


\section{Discussion}

Estimates, based on geometrical coverage of the photon detector plane, on the quantum effi-

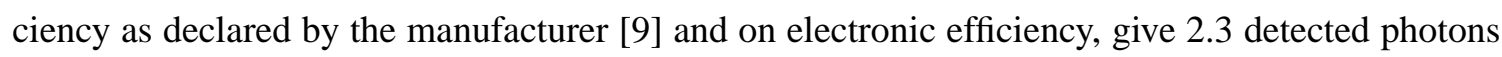
per charged particle, and our measured value is 1.6 photons/ring.

The increase in the signal-to-noise ratio obtained with the simple pyramidal light guides is also less than expected for the ideal case. Imperfections in the cutting and alignment of the light guides to the SiPMs, are possible causes. Further studies with different light guides may clarify the situation.

Finally, let us mention that an increase in the Cherenkov photon yield may be obtained by increasing the radiator thickness, refractive index and transparency. By making use of the focusing arrangement of aerogel radiators [i] $1 \overline{1}$, one may optimistically expect a factor of 5 increase in the radiator yield. Assuming that another factor of 2 could be obtained with improvement of light guide manufacture and alignment, one may expect that such a detector would meet the requirements for particle identification inside the upgraded Belle spectrometer.

\section{References}

[1] K. Abe et al. (edited by S. Hashimoto, M. Hazumi, J. Haba, J. W. Flanagan and Y. Ohnishi), Letter of Intent for KEK Super B Factory, KEK Report 2004-04 http : / / belle. kek . jp/superb

[2] I. Adachi et al., sBelle Design Study Report, arXiv:0810.4084 [hep-ex].

[3] P. Križan et al., Nucl. Instr. and Meth. A567 (2006) 124; S. Korpar et al., Nucl. Instr. and Meth. A595 (2008) 169.

[4] S. Nishida et al., Nucl. Instr. and Meth. A595 (2008) 161.

[5] P. Buzhan et al., An advanced study of silicon photomultiplier, ICFA Instrumentation Bulletin, Vol. 23 (2001).

[6] D. Renker, Nucl. Instr. and Meth. A567 (2006) 48.

[7] J. Haba, Nucl. Instr. and Meth. A595 (2008) 154.

[8] M. Danilov, Nucl. Instr. and Meth. A604 (2009) 183.

[9] MPPC data sheet, Hamamatsu Photonics.

[10] I. Ariño et al., Nucl. Instr. and Meth. A516 (2004) 445.

[11] T. Iijima, S. Korpar et al., Nucl. Instr. and Meth. A548 (2005) 383. 